\title{
Conjunction, Disjunction and Iterated Conditioning of Conditional Events
}

\author{
A. Gilio ${ }^{1}$ and G. Sanfilippo ${ }^{2}$
}

\begin{abstract}
Starting from a recent paper by S. Kaufmann, we introduce a notion of conjunction of two conditional events and then we analyze it in the setting of coherence. We give a representation of the conjoined conditional and we show that this new object is a conditional random quantity, whose set of possible values normally contains the probabilities assessed for the two conditional events. We examine some cases of logical dependencies, where the conjunction is a conditional event; moreover, we give the lower and upper bounds on the conjunction. We also examine an apparent paradox concerning stochastic independence which can actually be explained in terms of uncorrelation. We briefly introduce the notions of disjunction and iterated conditioning and we show that the usual probabilistic properties still hold.
\end{abstract}

Key words: Conditional events, conditional random quantities, conjunction, disjunction, iterated conditionals.

\section{Introduction}

In probability theory and in probability logic a relevant problem, largely discussed by many authors (see, e.g., $[2,3,7]$ ), is that of suitably defining logical operations among conditional events. In a recent paper by Kaufmann ([8]) a theory for the compounds of conditionals has been proposed. In this paper, based on the work of Kaufmann, we develop a similar theory in the framework of coherence. We show that conjunction and disjunction of conditional events

Dipartimento di Scienze di Base e Applicate per l'Ingegneria, University of Rome "La Sapienza", Italy, e-mail: angelo.gilio@sbai.uniroma1.it .

Dipartimento di Scienze Statistiche e Matematiche "S. Vianelli", University of Palermo, Italy, e-mail: giuseppe.sanfilippo@unipa.it 
in general are not conditional events but conditional random quantities. We give representations for such compounds and we study the coherent extensions of a probability assessment $(x, y)$ on two conditional events $\{A|H, B| K\}$ to their conjunction $(A \mid H) \wedge(B \mid K)$ and their disjunction $(A \mid H) \vee(B \mid K)$. In particular, by considering the conjunction, we show cases of logical dependencies in which the combination reduces to a conditional event. For reason of space we only give a short introduction to disjunction and iterated conditioning. We give the lower and upper bounds for conjunction, disjunction and iterated conditional and we show that the usual probabilistic properties still hold in terms of previsions. We also discuss an apparent paradox where $A|H, B| K$ seem to be stochastically independent, by giving an explanation in terms of uncorrelation between random quantities.

\section{Preliminary notions}

We denote events and their indicators by the same symbol and we write r.q. (resp., c.r.q.) for random quantity (resp., conditional random quantity). We recall that $n$ events are said logically independent when there are no logical dependencies among them, which amounts to say that the number of constituents is $2^{n}$. A conditional event $A \mid H$, where $H \neq \emptyset$, is a three-valued logical entity which is true, or false, or void, according to whether $A H$ is true, or $A^{c} H$ is true, or $H^{c}$ is true. In the setting of coherence, given an event $H \neq \emptyset$ and a finite r.q. $X \in\left\{x_{1}, x_{2}, \ldots, x_{n}\right\}$, agreeing to the betting metaphor the prevision $\mathbb{P}(X \mid H)$ of $X \mid H$ is defined as the amount $\mu$ you agree to pay, by knowing that you will receive the amount $X$ if $H$ is true, or you will receive back the amount $\mu$ if $H$ is false (bet called off). Then, still denoting by $X \mid H$ the amount that you receive, it holds that $X \mid H=X H+\mu H^{c}$ and, in what follows, based on the assessment $\mathbb{P}(X \mid H)=\mu$, we will look at the c.r.q. $X \mid H$ as the unconditional r.q. $X H+\mu H^{c}$. Operatively, what you pay is your prevision for $X \mid H$; then by linearity $\mathbb{P}(X \mid H)=\mu=\mathbb{P}\left(X H+\mu H^{c}\right)=$ $\mathbb{P}(X H)+\mu P\left(H^{c}\right)$, from which it follows $\mathbb{P}(X H)=P(H) \mu=P(H) \mathbb{P}(X \mid H)$. In particular, when $X$ is an event $A$, the prevision of $X \mid H$ is the probability of $A \mid H$ and, if you assess $P(A \mid H)=p$, then for the indicator of $A \mid H$, denoted by the same symbol, we have $A \mid H=A H+p H^{c} \in\{1,0, p\}$. Therefore $A \mid H \neq A$, but "conditionally on $H$ being true", i.e. for $H=1$, we have $A \mid H=A \in$ $\{1,0\}$, while $A \mid H=p$ for $H=0$.

Some authors look at the conditional "if $A$ then $C$ ", denoted $A \rightarrow C$, as the event $A^{c} \vee C$ (material conditional), but since some years it is becoming standard to look at $A \rightarrow C$ as the conditional event $C \mid A$ (see e.g. [4, 9]).

In [8], based on a complex procedure (which exploits the notion of Stalnaker Bernoulli space), by assuming $P(A)$ positive it is proved that $P(A \rightarrow C)=$ $\frac{P(A C)}{P(A)}=P(C \mid A)$. Then, by defining truth values of $A \rightarrow C$ (like conditional events) as: $V(A \rightarrow C)=1$, or 0 , or $P(C \mid A)$, according to whether $A C$ is true, 
or $A C^{c}$ is true, or $A^{c}$, is true, it is verified that the expectation of $V(A \rightarrow C)$ is $P(C \mid A)$. Moreover, assuming $P(A \vee C)>0$, Kaufmann obtains for the conjunction of $A \rightarrow B$ and $C \rightarrow D$ the formula

$$
P[(A \rightarrow B) \wedge(C \rightarrow D)]=\frac{P(A B C D)+P(B \mid A) P\left(A^{c} C D\right)+P(D \mid C) P\left(A B C^{c}\right)}{P(A \vee C)} .
$$

Based on this result, Kaufmann suggests a natural way of defining the values for the conjunction of conditionals. We will generalize the approach of Kaufmann in the setting of coherence in a direct and simpler way. Notice that in our paper the conjoined conditional will explicitly appear as a conditional random quantity; hence, we will speak of previsions (and not of probabilities).

\section{Conjunction of conditional events}

We preliminarily deepen an aspect of coherence and we exploit linearity of prevision to directly obtain the general compound prevision theorem. Given any event $H \neq \emptyset$ and any random quantities $X$ and $Y$, if $X H=Y H$ (that is, for $H=1$ it holds that $X|H=Y| H$ ), then coherence requires that $\mathbb{P}(X \mid H)=\mathbb{P}(X H \mid H)=\mathbb{P}(Y H \mid H)=\mathbb{P}(Y \mid H)$. In other words

$$
X H=Y H \Longrightarrow X\left|H=X H+\mathbb{P}(X \mid H) H^{c}=Y H+\mathbb{P}(Y \mid H) H^{c}=Y\right| H .
$$

Theorem 1. Given two events $H \neq \emptyset, K \neq \emptyset$ and a r.q. $X$, if the assessment $(x, y, z)$ on $\{H|K, X| H K, X H \mid K\}$ is coherent, then $z=x y$.

Proof. We have

$X \mid H K=X H K+y(H K)^{c}=X H K+y K^{c}+y H^{c} K=\left(X H+y H^{c}\right) K+y K^{c} ;$

moreover, by setting $\mathbb{P}\left[\left(X H+y H^{c}\right) \mid K\right]=\mu$, we have

$$
\left(X H+y H^{c}\right) \mid K=\left(X H+y H^{c}\right) K+\mu K^{c}=X H K+y H^{c} K+\mu K^{c} .
$$

As we can see: (i) $\left(X H+y H^{c}\right) \mid K=X H+y H^{c}$ when $K=1$; (ii) $(X H+$ $\left.y H^{c}\right) \mid K=\mu$ when $K=0$; moreover: (a) $X \mid H K=X H+y H^{c}$ when $K=1$; (b) $X \mid H K=y$ when $K=0$; that is, for $K=1$, both $X \mid H K$ and $(X H+$ $\left.y H^{c}\right) \mid K$ coincide with $X H+y H^{c}$. Then, by the same reasoning as in (1), by coherence $\mu$ must coincide with $y$ and by linearity of prevision:

$$
\mu=y=\mathbb{P}(X H \mid K)+y P\left(H^{c} \mid K\right)=z+y(1-x) ;
$$

hence: $z=x y$; that is: $\mathbb{P}(X H \mid K)=P(H \mid K) \mathbb{P}(X \mid H K)$.

We now introduce the notion of conjunction, by first giving some logical and probabilistic remarks. Given any events $A, B, H$, with $H \neq \emptyset$, let us 
consider the conjunction $A B$, or the conjunction $(A \mid H) \wedge(B \mid H)=A B \mid H$. We have: $A B=\min \{A, B\}=A \cdot B \in\{0,1\}$; moreover, if we assess $P(A \mid H)=$ $x, P(B \mid H)=y$, then $A\left|H=A H+x H^{c}, B\right| H=B H+y H^{c}$ and for $H=1$, i.e. conditionally on $H$ being true, we have:

$$
\begin{gathered}
A\left|H=A H+x H^{c}=A \in\{0,1\}, B\right| H=B H+y H^{c}=B \in\{0,1\}, \\
A B|H=\min \{A|H, B| H\}| H=\min \left\{A H+x H^{c}, B H+y H^{c}\right\} \mid H \in\{0,1\} .
\end{gathered}
$$

By defining $X=\min \{A|H, B| H\}=\min \left\{A H+x H^{c}, B H+y H^{c}\right\}$, we have $X \in\{1,0, x, y\}$ and, for $H=1, X|H=A B| H \in\{0,1\}$. Then, defining $\mathbb{P}(X \mid H)=\mu, P(A B \mid H)=z$, as in (1) by coherence $\mu=z$, so that for $H=0$ we have $X|H=A B| H=z$. In other words, $\min \{A|H, B| H\} \mid H$ and $A B \mid H$ are the same conditional random quantity. Then

$$
(A \mid H) \wedge(B \mid H)=\min \{A|H, B| H\}|H=\min \{A|H, B| H\}|(H \vee H) .
$$

In particular, for $B=A$, we have $A|H=(A \mid H)| H$, where $(A \mid H) \mid H$ is looked at as the c.r.q. $\left(A H+x H^{c}\right) \mid H$; this equality still holds from the viewpoint of iterated conditionals introduced in Section 6. Based on formula (2), we introduce below the notion of conjunction among conditional events.

Definition 1 (Conjunction). Given any pair of conditional events $A \mid H$ and $B \mid K$, with $P(A \mid H)=x, P(B \mid K)=y$, we define their conjunction as

$$
(A \mid H) \wedge(B \mid K)=\min \{A|H, B| K\}|(H \vee K)=(A \mid H) \cdot(B \mid K)|(H \vee K)
$$

Notice that, defining $Z=\min \{A|H, B| K\}$, the conjunction $(A \mid H) \wedge(B \mid K)$ is the c.r.q. $Z \mid(H \vee K)$. Moreover, defining $T=(A \mid H) \cdot(B \mid K)$, by coherence it holds that $Z|(H \vee K)=T|(H \vee K)$, while $Z \neq T$. Then,

$$
(A \mid H) \wedge(B \mid K)=[(A \mid H) \cdot(B \mid K)] \mid(H \vee K) .
$$

Interpretation with the betting scheme. If you assess $\mathbb{P}[(A \mid H) \wedge(B \mid K)]=z$, then you agree to pay the amount $z$ by receiving the amount $\min \{A|H, B| K\}$ if $H \vee K$ is true, or the amount $z$ if the bet is called off $(H \vee K$ false). That is, you pay $z$ and you receive the amount

$$
(A \mid H) \wedge(B \mid K)=\left\{\begin{array}{l}
1, \text { AHBK true } \\
0, A^{c} H \vee B^{c} K \text { true } \\
x, H^{c} B K \text { true } \\
y, A H K^{c} \text { true } \\
z, H^{c} K^{c} \text { true }
\end{array}\right.
$$

therefore, operatively, $(A \mid H) \wedge(B \mid K)$ can be represented as:

$$
(A \mid H) \wedge(B \mid K)=1 \cdot A H B K+x \cdot H^{c} B K+y \cdot A H K^{c}+z \cdot H^{c} K^{c} .
$$


Then, by linearity of prevision, it follows

$\mathbb{P}[(A \mid H) \wedge(B \mid K)]=z=P(A H B K)+x P\left(H^{c} B K\right)+y P\left(A H K^{c}\right)+z P\left(H^{c} K^{c}\right)$,

and we obtain $z P(H \vee K)=P(A H B K)+x P\left(H^{c} B K\right)+y P\left(A H K^{c}\right)$. In particular, if $P(H \vee K)>0$, we obtain the result of Kaufmann

$\mathbb{P}[(A \mid H) \wedge(B \mid K)]=\frac{P(A H B K)+P(A \mid H) P\left(H^{c} B K\right)+P(B \mid K) P\left(A H K^{c}\right)}{P(H \vee K)}$.

Some particular cases. We examine below the conjunction of $A \mid H$ and $B \mid K$ when there are some logical dependencies among $A, B, H, K$ and/or for special assessments $(x, y)$ on $\{A|H, B| K\}$. We set $P(A \mid H)=x, P(B \mid K)=$ $y, \mathbb{P}[(A \mid H) \wedge(B \mid K)]=z$.

1. If $x=y=1$, then $(A \mid H) \wedge(B \mid K)=1 \cdot A H B K+1 \cdot H^{c} B K+1 \cdot A H K^{c}+$ $+z \cdot H^{c} K^{c}=\left(A H \vee H^{c}\right) \wedge\left(B K \vee K^{c}\right) \mid(H \vee K)=\mathcal{C}(A|H, B| K)$, where $\mathcal{C}(A|H, B| K)$ is the quasi conjunction (see, e.g., $[3,5])$ of $A \mid H$ and $B \mid K$.

2. $K=A H$. As "conditionally on $H$ being true we have $(A \mid H) \wedge(B \mid A H)=$ $A B \mid H$ ", from (1) it follows

$$
(A \mid H) \wedge(B \mid A H)=1 \cdot A B H+z \cdot H^{c}=A B \mid H=\mathcal{C}(A|H, B| A H) .
$$

Then, by applying Theorem 1 to the family $\{A|H, B| A H, A B \mid H\}$, we have

$\mathbb{P}[(A \mid H) \wedge(B \mid A H)]=P(A B \mid H)=P(A \mid H) P(B \mid A H)=\mathbb{P}(A \mid H) \mathbb{P}(B \mid A H)$,

which means, as will see in Sec. 5, that $A \mid H$ and $B \mid A H$ are uncorrelated.

3. $A|H \subseteq B| K$, where $\subseteq$ denotes the inclusion relation of Goodman and Nguyen. In this case, coherence requires $x \leq y$; moreover, $A|H \leq B| K$, so that $\min \{A|H, B| K\}=A \mid H$. Then $A H K^{c}=\emptyset$ and we have

$$
(A \mid H) \wedge(B \mid K)=A H+x H^{c} B K+z H^{c} K^{c},
$$

from which it follows $z P(H \vee K)=x\left[P(H)+P\left(H^{c} B K\right)\right]$. By observing that $H B^{c} K=\emptyset$, we have $H \vee K=H \vee H^{c} K=H \vee H^{c} B K$; then $z P(H \vee$ $K)=x P(H \vee K)$, from which it follows $z=x$ if $P(H \vee K)>0$. Then, by the continuity property of coherence with respect to passages to the limits, the evaluation $z=x$ is coherent also for $P(H \vee K)=0$. By the methods of coherence, it can be shown that the extension $\mathbb{P}[(A \mid H) \wedge(B \mid K)]=z$ of the assessment $(x, 0)$ on $\{A \mid H, H \vee K\}$, where $A|H \subseteq B| K$, is coherent if and only if $z=x$. Then, from (3), as $H^{c} B^{c} K=\emptyset$ we obtain

$$
(A \mid H) \wedge(B \mid K)=A H+x\left(H^{c} B K+H^{c} B^{c} K+H^{c} K^{c}\right)=A\left|H+x H^{c}=A\right| H .
$$




\section{Lower and upper bounds for $(A \mid H) \wedge(B \mid K)$}

We will now determine the coherent extensions of the assessment $(x, y)$ on $\{A|H, B| K\}$ to their conjunction $(A \mid H) \wedge(B \mid K)$. We recall that the extension $z=P(A B \mid H)$ of the assessment $(x, y)$ on $\{A|H, B| H\}$, with $A, B, H$ logically independent, is coherent if and only if: $\max \{x+y-1,0\} \leq z \leq \min \{x, y\}$. The same results holds for $(A \mid H) \wedge(B \mid K)$ ! We have

Theorem 2. Given any coherent assessment $(x, y)$ on $\{A|H, B| K\}$, with $A, H, B, K$ logically independent, and with $H \neq \emptyset, K \neq \emptyset$, the extension $z=\mathbb{P}[(A \mid H) \wedge(B \mid K)]$ is coherent if and only if the Fréchet-Hoeffding bounds are satisfied, that is

$$
\max \{x+y-1,0\}=z^{\prime} \leq z \leq z^{\prime \prime}=\min \{x, y\} .
$$

For reasons of space we give the proof in the appendix; here we only give a sketch of the proof by the following steps:

1 ) by the logical independence of the events $A, H, B, K$, it can be verified that the assessment $(x, y)$ is coherent for every $(x, y) \in[0,1]^{2}$;

2 ) the values $z^{\prime}, z^{\prime \prime}$ are determined by studying the coherence of the assessment $\mathcal{P}=(x, y, z)$ on $\mathcal{F}=\{A|H, B| K,(A \mid H) \wedge(B \mid K)\}$, by means of a geometrical approach (see, e.g., [5]);

3 ) the points associated with the constituents generated by $\mathcal{F}$ and contained in $H \vee K$ are: $Q_{1}=(1,1,1), Q_{2}=(1,0,0), Q_{3}=(0,1,0), Q_{4}=(0,0,0)$, $Q_{5}=(1, y, y), Q_{6}=(0, y, 0), Q_{7}=(x, 1, x), Q_{8}=(x, 0,0)$;

$3)$ we consider the convex hull $\mathcal{I}$ of $Q_{1}, \ldots, Q_{8}$; then, we study the solvability of the linear system representing the condition $\mathcal{P} \in \mathcal{I}$, which is necessary, and in our case also sufficient, for the coherence of $\mathcal{P}$;

4) finally we obtain that, for any given pair $(x, y) \in[0,1]^{2}$, the assessment $\mathcal{P}$ is coherent if and only if $\max \{x+y-1,0\}=z^{\prime} \leq z \leq z^{\prime \prime}=\min \{x, y\}$; i.e.

$$
\max \{P(A \mid H)+P(B \mid K)-1,0\} \leq \mathbb{P}[(A \mid H) \wedge(B \mid K)] \leq \min \{P(A \mid H), P(B \mid K)\} .
$$

We remark that for quasi conjunction the inequalities (4) do not hold; indeed, the extension $\gamma=P[\mathcal{C}(A|H, B| K)]$ of the assessment $(x, y)$ is coherent if and only if $\gamma^{\prime} \leq \gamma \leq \gamma^{\prime \prime}$, where $\gamma^{\prime}=z^{\prime}=\max \{x+y-1,0\}$ and $\gamma^{\prime \prime}=S_{0}^{H}(x, y)$, where $S_{0}^{H}(x, y)=\frac{x+y-2 x y}{1-x y}$ if $(x, y) \neq(1,1), S_{0}^{H}(x, y)=1$ if $(x, y)=(1,1)$ (Hamacher t-conorm). We observe that: $\gamma^{\prime \prime} \geq \max \{x, y\} \geq \min \{x, y\}=z^{\prime \prime}$.

\section{An apparent paradox on $(A \mid H) \wedge(B \mid K)$}

In this section ${ }^{1}$ we consider the case $H K=\emptyset$, where it seems that $A \mid H$ and $B \mid K$ are stochastically independent; this appears unreasonable; is it? Actually, assuming $H K=\emptyset$, the constituents contained in $H \vee K$ are $C_{1}=A H K^{c}, C_{2}=A^{c} H K^{c}, C_{3}=H^{c} B K, C_{4}=H^{c} B^{c} K$ and, given

\footnotetext{
1 The study of this case was stimulated by a discussion between D Edgington and A Gilio.
} 
the assessment $P(A \mid H)=x, P(B \mid K)=y, \mathbb{P}[(A \mid H) \wedge(B \mid K)]=z$, the associated vectors of numerical values for $A|H, B| K,(A \mid H) \wedge(B \mid K)$ are $Q_{1}=(1, y, y), Q_{2}=(0, y, 0), Q_{3}=(x, 1, x), Q_{4}=(x, 0,0)$. Let $\mathcal{I}$ be the convex hull of $Q_{1}, \ldots, Q_{4}$. In our case, the condition $\mathcal{P} \in \mathcal{I}$, which is necessary for the coherence of $\mathcal{P}$, is also sufficient and after some computation on the associated linear system it can be verified that $\mathcal{P}$ is coherent if and only if $z=x y$ and $(x, y) \in[0,1]^{2}$. Therefore, coherence requires that

$$
\mathbb{P}[(A \mid H) \wedge(B \mid K)]=P(A \mid H) P(B \mid K)=\mathbb{P}(A \mid H) \mathbb{P}(B \mid K) .
$$

Does (5) mean that $A \mid H$ and $B \mid K$ are stochastically independent? The answer, as shown below, is negative. Indeed, we observe that:

(i) by Definition $1,(A \mid H) \wedge(B \mid K)=(A \mid H) \cdot(B \mid K) \mid(H \vee K)$ is a conditional random quantity, not a conditional event; then the correct framework for giving a meaning to equality (5) is that of random quantities; moreover, in our case we have: $(A \mid H) \wedge(B \mid K)=x H^{c} B K+y A H K^{c}+z H^{c} K^{c}$;

(ii) $(A \mid H) \cdot(B \mid K)=\left(A H+x H^{c}\right)\left(B K+y K^{c}\right)=x H^{c} B K+y A H K^{c}+x y H^{c} K^{c}$; (iii) as $z=x y$, we have $(A \mid H) \wedge(B \mid K)=(A \mid H) \cdot(B \mid K)$; that is, the conjunction is the product of the conditional random quantities $A|H, B| K$.

Then, (5) only means that $A \mid H$ and $B \mid K$ are uncorrelated, and does not mean that they are independent. Hence, by the previous reasoning we have proved

Theorem 3. Given any events $A, B, H, K$, with $H \neq \emptyset, K \neq \emptyset, H K=\emptyset$, it holds that $\mathbb{P}[(A \mid H) \cdot(B \mid K)]=\mathbb{P}(A \mid H) \mathbb{P}(B \mid K)$; that is, the random quantities $A \mid H$ and $B \mid K$ are uncorrelated.

We remark that, as shown in case 2, Section 3, where $B=A H, A \mid H$ and $B \mid K$ could be uncorrelated even if $H K \neq \emptyset$. Indeed, by formula (1), we have

$$
(A \mid H) \cdot(B \mid A H)=A B H+x y \cdot H^{c}=A B H+z \cdot H^{c}=(A \mid H) \wedge(B \mid A H),
$$

and then $\mathbb{P}[(A \mid H) \cdot(B \mid A H)]=\mathbb{P}[(A \mid H) \wedge(B \mid A H)]=\mathbb{P}(A \mid H) \mathbb{P}(B \mid A H)$.

\section{Disjunction and iterated conditioning}

We define below the notions of disjunction and of iterated conditioning; in [6] we are working on an expanded version of this paper. A notion of conditioning among random quantities has been studied in [1].

Definition 2 (Disjunction). Given any pair of conditional events $A \mid H$ and $B \mid K$, we define $(A \mid H) \vee(B \mid K)=\max \{A|H, B| K\} \mid(H \vee K)$.

By assessing $P(A \mid H)=x, P(B \mid K)=y, \mathbb{P}[(A \mid H) \vee(B \mid K)]=\gamma$, we have $(A \mid H) \vee(B \mid K)=1 \cdot(A H \vee B K)+x \cdot H^{c} B^{c} K+y \cdot A^{c} H K^{c}+\gamma \cdot H^{c} K^{c}$. By coherence, it can be proved that the prevision sum rule holds, that is $\mathbb{P}[(A \mid H) \vee(B \mid K)]=\mathbb{P}(A \mid H)+\mathbb{P}(B \mid K)-\mathbb{P}[(A \mid H) \wedge(B \mid K)]$, and from (4) 
$\max \{P(A \mid H), P(B \mid K)\} \leq \mathbb{P}[(A \mid H) \vee(B \mid K)] \leq \min \{P(A \mid H)+P(B \mid K)-1,1\}$

Definition 3 (Iterated conditioning). Given any pair of conditional events $A \mid H$ and $B \mid K$ we define the iterated conditional $(B \mid K) \mid(A \mid H)$ as

$$
(B \mid K)\left|(A \mid H)=(B \mid K) \wedge(A \mid H)+\mu A^{c}\right| H,
$$

where $\mu$ is the prevision of $(B \mid K) \mid(A \mid H)$ and represents the amount you agree to pay, with the proviso that you will receive the quantity $(B \mid K) \mid(A \mid H)$.

If $P(A \mid H)=x, P(B \mid K)=y, \mathbb{P}[(A \mid H) \wedge(B \mid K)]=z$, the values of $(B \mid K) \mid(A \mid H)$ are $1,0, y, \mu, x+\mu(1-x), \mu(1-x), z+\mu(1-x)$, respectively associated with the constituents $A H B K, A H B^{c} K, A H K^{c}, A^{c} H, H^{c} B K, H^{c} B^{c} K, H^{c} K^{c}$. By linearity of prevision: $\mathbb{P}[(B \mid K) \mid(A \mid H)]=\mu=\mathbb{P}[(B \mid K) \wedge(A \mid H)]+\mu P\left(A^{c} \mid H\right)$; that is: $\mu=z+\mu(1-x)$, from which it follows

$$
\mathbb{P}[(B \mid K) \wedge(A \mid H)]=\mathbb{P}[(B \mid K) \mid(A \mid H)] P(A \mid H) .
$$

Then, assuming $x=P(A \mid H)>0, P(H \vee K)>0$, one has: $\mathbb{P}[(B \mid K) \mid(A \mid H)]=$ $\mu=\frac{\mathbb{P}[(B \mid K) \wedge(A \mid H)]}{P(A \mid H)}=\frac{z}{x}=\frac{P(A H B K)+P(A \mid H) P\left(H^{c} B K\right)+P(B \mid K) P\left(A H K^{c}\right)}{P(A \mid H) P(H \vee K)}$, which coincides with the result of Kaufmann. If we only assign $x$ and $y$, then $\max \{0, x+y-1\} \leq z \leq \min \{x, y\}$, and it follows $\mu \in\left[\mu^{\prime}, \mu^{\prime \prime}\right]$, with $\mu^{\prime}=0$, $\mu^{\prime \prime}=1$ for $x=0$, and with $\mu^{\prime}=\max \left\{0, \frac{x+y-1}{x}\right\}, \mu^{\prime \prime}=\min \left\{1, \frac{y}{x}\right\}$ for $x>0$.

Acknowledgements We thank the anonymous referees for their useful comments.

\section{References}

1. Biazzo V, Gilio A, Sanfilippo G (2009) On general conditional prevision assessments. Proc. WUPES'09, Liblice, Czech Rep., Sept. 19-23, 2009, pp 23-34

2. Capotorti A, Vantaggi B (1999) A general interpretation of conditioning and its implication on coherence, Soft Computing, 3:148-153

3. Dubois D, Prade H (1994) Conditional Objects as Nonmonotonic Consequence relationships. IEEE Trans Syst Man Cybern 24:1724-1740

4. Gilio A, Over D (2012) The psychology of inferring conditionals from disjunctions: A probabilistic study. Journal of Mathematical Psychology, 56(2):118-131

5. Gilio A, Sanfilippo G (2011) Quasi Conjunction and Inclusion Relation in Probabilistic Default Reasoning. In: Liu W (ed) Symbolic and Quantitative Approaches to Reasoning with Uncertainty, LNCS, vol 6717, Springer Berlin Heidelberg, pp 497-508

6. Gilio A, Sanfilippo G (2012) A Coherence-Based Probabilistic Theory of Compounds of Conditionals. Working paper; presented at the Workshop "Conditionals, Counterfactuals and Causes in Uncertain Environments", Düsseldorf, May 19-22, 2011. Slides available at http://www.phil-fak.uni-duesseldorf.de/conditionals/

7. Goodman IR, Nguyen HT, Walker EA (1991) Conditional Inference and Logic for Intelligent Systems: A Theory of Measure-free Conditioning, North-Holland

8. Kaufmann S (2009) Conditionals Right and Left: Probabilities for the Whole Family, J Philos Logic 38:1-53 
9. Pfeifer N, Kleiter GD (2010) The conditional in mental probability logic. In Oaksford M, Chater N (eds), Cognition and conditionals: Probability and logic in human thought. Oxford University Press, pp 153-173 